\title{
Efficacy and Safety of Crisaborole Ointment, 2\%, for the Treatment of Mild-to-Moderate Atopic Dermatitis Across Racial and Ethnic Groups
}

\author{
Valerie D. Callender ${ }^{1,2} \circledast$ - Andrew F. Alexis ${ }^{3,4} \cdot$ Linda F. Stein Gold ${ }^{5} \cdot$ Mark G. Lebwohl $^{4} \cdot$ Amy S. Paller $^{6}$. \\ Seemal R. Desai ${ }^{7,8} \cdot$ Huaming Tan $^{9} \cdot$ William C. Ports $^{9} \cdot$ Michael A. Zielinski $^{10} \cdot$ Anna M. Tallman ${ }^{11}$
}

Published online: 1 July 2019

(c) The Author(s) 2019

\begin{abstract}
Background Atopic dermatitis is highly prevalent in black/African American, Asian, and Hispanic patients, making assessment of these populations in clinical trials important. Crisaborole ointment, $2 \%$, is a nonsteroidal phosphodiesterase 4 inhibitor for the treatment of mild-to-moderate atopic dermatitis. In two pivotal phase III clinical trials in patients aged $\geq 2$ years, crisaborole was superior to vehicle in reducing global disease severity. The most common treatment-related adverse event was application site pain.

Objective The objective of this study was to investigate the efficacy and safety of crisaborole according to patient race and ethnicity.

Methods A pooled post hoc analysis by race and ethnicity of the two pivotal trials and a safety extension trial was performed. Race included white or nonwhite (encompassing Asian/native Hawaiian/other Pacific Islander, black/African American, and other/American Indian/Alaskan native); ethnicity included Hispanic/Latino or not Hispanic/Latino.

Results In white, nonwhite, Hispanic/Latino, and not Hispanic/Latino groups at day 29, more crisaborole- than vehicletreated patients achieved improvements in global disease severity [Investigator's Static Global Assessment of clear/almost clear with a $\geq 2$-grade improvement (white: $33.5 \%$ vs. $22.3 \%$, nominal $p<0.001$; nonwhite: $30.0 \%$ vs. $21.3 \%$, nominal $p<0.05$; Hispanic/Latino: $35.4 \%$ vs. $18.2 \%$, nominal $p<0.01$; not Hispanic/Latino: $31.3 \%$ vs. $22.8 \%$, nominal $p<0.01$ )]. Crisaborole treatment also improved atopic dermatitis signs/symptoms and quality of life. Frequency of crisaborole-related adverse events was $7.1-8.5 \%$ in the pivotal trials.

Conclusion Across races and ethnicities, crisaborole demonstrated efficacy for the treatment of mild-to-moderate atopic dermatitis, with a low frequency of treatment-related adverse events.
\end{abstract}

Anna M. Tallman: Former Pfizer employee.

Electronic supplementary material The online version of this article (https://doi.org/10.1007/s40257-019-00450-w) contains supplementary material, which is available to authorized users.

Valerie D. Callender

drcallender@CallenderSkin.com

1 Howard University College of Medicine, Washington, DC, USA

2 Callender Dermatology and Cosmetic Center, Glenn Dale, MD, USA

3 Mount Sinai St. Luke's and Mount Sinai West, New York, NY, USA

4 Icahn School of Medicine at Mount Sinai, New York, NY, USA
5 Henry Ford Health System, Detroit, MI, USA

6 Northwestern University Feinberg School of Medicine, Chicago, IL, USA

7 University of Texas Southwestern Medical Center, Dallas, TX, USA

Innovative Dermatology, Plano, TX, USA

9 Pfizer Inc., Groton, CT, USA

10 Pfizer Inc., Collegeville, PA, USA

11 Pfizer Inc., New York, NY, USA 


\section{Key Points}

Although the prevalence and presentation of atopic dermatitis (AD) vary by race and ethnicity, existing treatment guidelines do not provide specific recommendations for treating patients with skin of color.

Crisaborole improved global disease severity in patients aged $\geq 2$ years with mild-to-moderate $\mathrm{AD}$ across races and ethnicities, with a low frequency of treatment-related adverse events.

No adverse events related to skin discoloration were reported with crisaborole treatment in nonwhite and Hispanic/Latino patients, and crisaborole improved AD signs/symptoms common in these populations, including induration/papulation and lichenification.

\section{Introduction}

Atopic dermatitis (AD) is a common inflammatory skin disorder that often follows a chronic relapsing course [1, 2]. AD is characterized by inflamed eczematous lesions and intense pruritus [2]. Mounting evidence suggests that $\mathrm{AD}$ epidemiology, etiology, and presentation vary across racial and ethnic groups. National surveys as well as smaller studies conducted in US general pediatric and pediatric dermatology clinics have reported a higher AD prevalence in children who are black/African American [3-5], Asian [5, 6], and Hispanic [5] compared with white/not Hispanic children. Global trends provide further evidence of racial/ ethnic disparities, as phase III of the International Study of Asthma and Allergies in Childhood (ISAAC) reported wide global variation in eczema prevalence, with prevalence rates for children aged 6-7 years ranging from 0.9\% (Jodhpur, India) to 22.5\% (Quito, Ecuador) [7]. Disease burden may be higher in patients with skin of color, as black/African American and Asian children are more likely than white children to visit a doctor for a diagnosis of $\mathrm{AD}[5,8]$. Patients with skin of color may also progress to greater disease severity before diagnosis as a result of racial and ethnic variations in presentation and difficulty in discerning AD signs, such as erythema $[9,10]$. Disease presentation varies by race and ethnicity in that the dermatitic lesions of patients with skin of color are more likely to be follicular based, papular, or lichenoid, and resolved lesions tend to show dyschromia/ altered pigmentation $[9,11]$. Compared with those of European Americans, lesions of East Asian individuals are more likely to be psoriasiform and to show clear demarcation, lichenification, and scaling [12, 13]. African Americans are more likely than other racial groups to present with prurigo nodularis, lichenification, scattered follicular papules on the extensors and trunk, and lichen planus-like $\operatorname{AD}[9,13]$.

Immune mechanisms may contribute to ethnic and racial variation in $\mathrm{AD}$ presentation. Compared with $\mathrm{AD}$ lesions of European Americans, lesions of East Asian individuals show a greater induction of T-helper (Th) 17 and Th22 cytokines [12], and lesions of African Americans show reduced activation of Th1 and Th17 axes [14]. Other hypothesized racial immune differences include higher serum immunoglobulin E levels and larger mast cell granule size in black/African American patients compared with white patients [9, 14-16].

Despite noted racial and ethnic differences in disease presentation and immune profile, patients with skin of color are underrepresented in clinical trials for $\mathrm{AD}$, and race and ethnicity are often not considered in the interpretation of findings [17]. Reflecting these patterns, treatment guidelines do not provide specific information for treating patients with skin of color [18-20]. As AD is more prevalent in patients with skin of color, racial and ethnic subgroup analyses of clinical trial data are of particular importance [17].

Crisaborole ointment is a nonsteroidal phosphodiesterase 4 inhibitor for the treatment of mild-to-moderate AD [21]. Inhibition of phosphodiesterase 4 by crisaborole increases levels of 3'5'-cyclic adenosine monophosphate in inflammatory cells, leading to activation of nuclear factor $\kappa \mathrm{B}$ and nuclear factor of activated T-cell signaling pathways and subsequent suppression of inflammatory cytokine release. Two identically designed, pivotal phase III trials investigating the safety and efficacy of crisaborole ointment, $2 \%$, for patients with mild-to-moderate AD found that more crisaborole-treated patients than vehicle-treated patients achieved improvement in global disease severity and signs and symptoms of $\mathrm{AD}$, including pruritus [22]. Most treatment-emergent adverse events (TEAEs) were mild-to-moderate and considered unrelated to treatment. Long-term safety analysis for crisaborole from the two pivotal studies and a 48-week safety extension study showed a low frequency of treatmentrelated adverse events (AEs) $(10.3 \%)$, of which the most frequently reported were $\mathrm{AD}[3.1 \%$; worsening, exacerbation, flare, or flare-up of existing condition, classified by the Medical Dictionary for Regulatory Activities (MedDRA) as dermatitis atopic], application site pain (2.3\%), and application site infection (1.2\%) [23].

The objective of this pooled post hoc analysis of the pivotal studies and long-term safety study was to investigate the efficacy and safety of crisaborole ointment, $2 \%$, for the treatment of mild-to-moderate $\mathrm{AD}$ in patients aged $\geq 2$ years according to race and ethnicity. 


\section{Methods}

\subsection{Study Design and Oversight}

A pooled post hoc analysis of two identically designed, multicenter, randomized, vehicle-controlled, double-blind, pivotal phase III studies (ClinicalTrials.gov AD-301: NCT02118766; AD-302: NCT02118792) and an open-label, 48-week, long-term safety study (AD-303) was conducted. Full details of the phase III and long-term safety studies have been reported previously $[22,23]$.

\subsection{Patients}

In the two pivotal studies (AD-301, AD-302), patients were randomly assigned 2:1 to receive crisaborole ointment or vehicle. Key inclusion criteria required patients to be aged $\geq 2$ years, have a clinical diagnosis of $A D$ according to Hanifin and Rajka criteria, have an Investigator's Static Global Assessment (ISGA) score of mild (2) or moderate (3) at baseline, and have $\geq 5 \%$ treatable body surface area involvement (excluding the scalp). At selected investigator sites, patients completing pivotal study AD-301 or AD-302 without experiencing a treatment-related $\mathrm{AE}$ or serious $\mathrm{AE}$ that prompted crisaborole discontinuation were eligible for enrollment in the long-term safety extension study (AD-303) within 8 days of the end of the study visit.

\subsection{Crisaborole or Vehicle Treatment}

In the two pivotal studies (AD-301, AD-302), patients were instructed to apply a thin layer of study drug ointment (crisaborole or vehicle) twice daily for 28 days to all treatable AD-involved areas (excluding the scalp) identified at baseline/day 1 and to any new lesions appearing after day 1 . In the long-term extension study (AD-303), patient global disease severity was assessed every 28 days for 48 weeks using the ISGA, a 5-point static scale indicating clear (0), almost clear (1), mild (2), moderate (3), or severe (4) disease. Patients with ISGA scores $\geq 2$ entered or continued an additional 28-day on-treatment period of twice-daily crisaborole application. Patients with ISGA scores $\leq 1$ entered or continued an additional off-treatment period during which no study drug was applied. Crisaborole ointment treatment was discontinued in patients who showed no improvement in ISGA score after three consecutive on-treatment periods. The maximum number of consecutive treatment cycles was 3. In all three trials, the use of bland emollient(s) to manage dry skin was permitted on uninvolved skin but prohibited on AD-involved skin.

\subsection{Efficacy Assessments (AD-301, AD-302)}

Global disease severity was assessed at baseline and on days $8,15,22$, and 29 using the ISGA scale. The primary efficacy endpoint was the proportion of patients achieving success in ISGA at day 29 in the crisaborole-treated group compared with the vehicle-treated group. Success in ISGA was defined as an ISGA score of clear (0) or almost clear (1) with a $\geq 2$-grade improvement from baseline. The secondary efficacy endpoints were the proportion of patients with ISGA scores of clear (0) or almost clear (1) at day 29 and time to ISGA success. Additional efficacy endpoints included improvements in pruritus, signs of $\mathrm{AD}$, and quality of life (QoL). Pruritus was measured twice daily by an electronic diary using the Severity of Pruritus (SPS) scale, a 4-point scale indicating none (0), mild (1), moderate (2), or severe (3) pruritus. Baseline pruritus severity was calculated as the average of two or more assessments on day 1. Weekly SPS scores were calculated for each patient as the average of all available post-baseline SPS scores during the corresponding week (generally up to 14 measurements). Signs of AD (erythema, induration/papulation, exudation, excoriation, and lichenification) were evaluated at baseline and on days 8 , 15,22 , and 29 using a 4-point scale from none (0) to severe (3). Improvement in pruritus or AD signs was defined as a score of none ( 0 ) or mild (1) with $\mathrm{a} \geq 1$-grade improvement from baseline. QoL assessments were made at baseline and at day 29 using age-appropriate questionnaires [Children's Dermatology Life Quality Index (CDLQI) for patients aged 2-15 years; Dermatology Life Quality Index (DLQI) for patients aged $\geq 16$ years; Dermatology Family Impact Questionnaire (DFI) for parents/caregivers/families of patients aged 2-17 years]. All QoL assessments were made on a scale of 0-30 points, with higher scores indicating poorer QoL. In study AD-303, there were no pre-defined efficacy analyses, as this was an open-label, long-term, safety extension study.

\subsection{Safety Assessments (AD-301, AD-302, AD-303)}

Frequencies of AEs and serious AEs (SAEs) were assessed at baseline, at scheduled and unscheduled investigator visits, and at study conclusion in the pivotal studies (AD-301, AD-302). Frequencies of AEs and SAEs and local tolerability were assessed on the first day of each 28-day treatment cycle and at study conclusion for the long-term extension study (AD-303). AEs were recorded and classified using MedDRA terminology. TEAEs were defined as AEs with an onset on or after the day of the first study drug dose. Treatment-related TEAEs were events determined by the study investigator to be definitely, probably, or possibly related to treatment. 


\subsection{Post Hoc Statistical Analysis}

Efficacy and safety endpoints were assessed by racial and ethnic group. For statistical analyses, the designated racial categories were white and nonwhite, with the nonwhite group encompassing the following racial subgroups: Asian/ native Hawaiian/other Pacific Islander, black/African American, and other/American Indian/Alaskan native. Designated ethnic categories were Hispanic/Latino and not Hispanic/ Latino. Efficacy analyses were performed on the intentionto-treat (ITT) population, defined as all patients who were randomly assigned and dispensed the study drug. For ISGA, the difference between treatment groups in the proportions of patients achieving predefined improvement or successful outcomes was calculated at days $8,15,22$, and 29. Time to ISGA success was plotted using Kaplan-Meier methods. For signs of $\mathrm{AD}$ at day 29, the difference between treatment groups in the proportion of patients achieving improvement was calculated. For QoL scores at day 29, the difference between treatment groups in the least-squares mean change from baseline was calculated. Post hoc analysis of the difference in the proportion of patients achieving improvement in pruritus at weeks 1, 2, 3, and 4 was performed as described previously [24]. Statistical significance was set at a nominal 0.05 level. Nonwhite subgroups (Asian/native
Hawaiian/other Pacific Islander, black/African American, and other/American Indian/Alaskan native) were small and were therefore pooled to improve reliability of statistical analyses. $p$ values for the nonwhite subgroups are provided for informational purposes, but interpretation of these values is limited by the small sample size. The safety population consisted of all patients who were randomized, received one or more confirmed doses of the study drug, and had one or more post-baseline assessments. For safety endpoints in the pivotal (AD-301 and AD-302) and long-term extension (AD-303) studies, frequencies of TEAEs, serious TEAEs, and treatment-related TEAEs were summarized. Long-term safety data include AEs reported throughout the pivotal studies (AD-301 and AD-302, 4 weeks) and the long-term safety study (AD-303, 48 weeks) for a total of 52 weeks among patients who enrolled in study AD-303.

\section{Results}

\subsection{Patients and Enrollment}

The ITT population of the pivotal studies (AD-301 and AD-302) included a total of 1522 patients assigned 1016:506 to receive crisaborole:vehicle [Table 1 , white $(N=923)$,

Table 1 Baseline demographics and disease characteristics [AD-301 and AD-302 intention-to-treat (ITT) population]

\begin{tabular}{|c|c|c|c|c|c|c|c|c|}
\hline & \multicolumn{2}{|l|}{ White } & \multicolumn{2}{|l|}{ Nonwhite } & \multicolumn{2}{|c|}{ Hispanic/Latino } & \multicolumn{2}{|c|}{ Not Hispanic/Latino } \\
\hline & $\begin{array}{l}\text { Crisaborole } \\
N=617\end{array}$ & $\begin{array}{l}\text { Vehicle } \\
N=306\end{array}$ & $\begin{array}{l}\text { Crisaborole } \\
N=399\end{array}$ & $\begin{array}{l}\text { Vehicle } \\
N=200\end{array}$ & $\begin{array}{l}\text { Crisaborole } \\
N=200\end{array}$ & $\begin{array}{l}\text { Vehicle } \\
N=101\end{array}$ & $\begin{array}{l}\text { Crisaborole } \\
N=816\end{array}$ & $\begin{array}{l}\text { Vehicle } \\
N=405\end{array}$ \\
\hline $\begin{array}{l}\text { Age, mean } \\
\text { (range), } \\
\text { years }\end{array}$ & $12.3(2-79)$ & $11.2(2-79)$ & $12.3(2-64)$ & $13.4(2-72)$ & $10.5(2-59)$ & $9.2(2-41)$ & $12.8(2-79)$ & $12.8(2-79)$ \\
\hline \multicolumn{9}{|c|}{ Age group, $\%$, years } \\
\hline $2-11$ & 62.2 & 63.7 & 60.9 & 60.0 & 63.0 & 68.3 & 61.4 & 60.7 \\
\hline $12-17$ & 24.6 & 25.5 & 23.8 & 23.0 & 28.0 & 23.8 & 23.4 & 24.7 \\
\hline$\geq 18$ & 13.1 & 10.8 & 15.3 & 17.0 & 9.0 & 7.9 & 15.2 & 14.6 \\
\hline Female, $\%$ & 51.7 & 51.6 & 61.9 & 61.5 & 52.0 & 60.4 & 56.6 & 54.3 \\
\hline \multicolumn{9}{|l|}{ ISGA, \% } \\
\hline Mild: 2 & 37.8 & 37.6 & 40.1 & 39.0 & 37.0 & 34.7 & 39.1 & 39.0 \\
\hline Moderate: 3 & 62.2 & 62.4 & 59.9 & 61.0 & 63.0 & 65.3 & 60.9 & 61.0 \\
\hline \multicolumn{9}{|c|}{ Average SPS score, $\%$} \\
\hline $\begin{array}{l}\text { None: } 0 \\
\quad \text { to }<0.5\end{array}$ & 1.62 & 1.63 & 1.25 & 1.00 & 2.50 & 2.97 & 1.23 & 0.99 \\
\hline $\begin{array}{r}\text { Mild: } 0.5 \\
\text { to }<1.5\end{array}$ & 17.99 & 18.95 & 18.05 & 23.00 & 17.50 & 17.82 & 18.14 & 21.23 \\
\hline $\begin{array}{l}\text { Moderate: } \\
1.5 \text { to }<2.5\end{array}$ & 36.63 & 32.68 & 31.58 & 31.00 & 36.00 & 33.66 & 34.31 & 31.60 \\
\hline $\begin{array}{l}\text { Severe: } 2.5 \\
\text { to } \leq 3\end{array}$ & 20.58 & 19.61 & 21.80 & 19.00 & 21.00 & 20.79 & 21.08 & 19.01 \\
\hline
\end{tabular}

The ITT population was defined as all patients who were randomly assigned and dispensed the study drug

ISGA Investigator's Static Global Assessment, SPS severity of pruritus 
nonwhite $(N=599)$, Hispanic/Latino $(N=301)$, not Hispanic/Latino $(N=1221)]$. Baseline demographics and disease characteristics were similar across treatment and race/ ethnicity groups. The proportion of patients with mild (ISGA 2, 34.7-40.1\%) or moderate (ISGA 3, 59.9-65.3\%) AD at baseline was similar across race, ethnicity, and treatment groups, and the majority of patients had moderate (average SPS 1.5 to $<2.5,31.0-36.63 \%$ ) and severe (average SPS 2.5 to $\leq 3,19.0-21.8 \%$ ) pruritus at baseline. Within the nonwhite group, 424 patients (70.8\%) were black/African American, 94 patients (15.7\%) were Asian/native Hawaiian/other Pacific Islander, and 81 patients (13.5\%) were other/American Indian/Alaskan native [see Table 1 of the Electronic Supplementary Material (ESM) for baseline demographics and disease characteristics of nonwhite racial subgroups].

\subsection{Efficacy Endpoints}

More crisaborole-treated patients than vehicle-treated patients achieved success in ISGA on days $8,15,22$, and 29 (the primary efficacy endpoint) across race and ethnicity (AD-301 and AD-302, nominal $p<0.05$ for all racial and ethnic groups and time points) (Fig. 1a, b). The proportion of patients achieving success in ISGA at day 29 in crisaborole-treated vs. vehicle-treated patients in nonwhite racial subgroups was $20.6 \%$ vs. $14.2 \%$ (Asian/native Hawaiian/ other Pacific Islander, nominal $p=0.4524), 32.1 \%$ vs. $24.6 \%$ (black/African American, nominal $p=0.1349$ ), and 29.1\% vs. $13.2 \%$ (other/American Indian/Alaskan native, nominal $p=0.0923$ ). Shorter time to ISGA success with crisaborole vs. vehicle was observed in white, nonwhite, Hispanic/ Latino, and not Hispanic/Latino groups, and a similar pattern was observed for nonwhite racial subgroups (Figs. 1, 2 of the ESM).

More crisaborole-treated than vehicle-treated patients also achieved ISGA scores of clear or almost clear at all time points in all racial and ethnic groups (nominal $p<0.05$, Fig. 2a, b). Proportions of patients achieving clear or almost clear ISGA scores at day 29 in nonwhite racial subgroups (crisaborole vs. vehicle) was $33.8 \%$ vs. $28.5 \%$ (Asian/native Hawaiian/other Pacific Islander, nominal $p=0.6152), 49.7 \%$ vs. 33.7\% (black/African American, nominal $p<0.01$ ), and $54.9 \%$ vs. $34.5 \%$ (other/American Indian/Alaskan native, nominal $p=0.0904)$.

A greater proportion of crisaborole-treated patients than vehicle-treated patients achieved improvement in pruritus at weeks 1, 2, 3, and 4 for all racial and ethnic groups (nominal $p<0.05$, Fig. 3a). Proportions of patients achieving improvement in pruritus at week 4 in nonwhite racial subgroups (crisaborole vs. vehicle) was $28.6 \%$ vs. $9.5 \%$ (Asian/native Hawaiian/other Pacific Islander, nominal $p<0.05), 33.5 \%$ vs. $24.7 \%$ (black/African American, nominal $p=0.1199$ ), and $32.6 \%$ vs. $15.0 \%$ (other/American Indian/Alaskan native, nominal $p=0.1013$ ).

At day 29, more crisaborole- than vehicle-treated patients achieved improvement in most AD signs across race and ethnicity groups (Fig. 3b). A significant difference (nominal $p<0.05$ ) in proportions of patients with improvement in erythema, excoriation, and lichenification at day 29 in crisaborole-treated vs. vehicle-treated patients was observed in all race and ethnicity groups. More crisaborole-treated than vehicle-treated patients demonstrated improvement in lichenification, an AD sign often observed in patients with skin of color, at days 8 and 15 in all groups (nominal $p<0.05$, Table 2 of the ESM). Proportions of patients achieving improvement in lichenification remained greater through day 29 in the white, Hispanic/Latino, and not Hispanic/Latino groups (nominal $p<0.05$ ) and was numerically greater in the nonwhite group through day 29 (nominal $p<0.06$ ). Table 3 of the ESM reports data for nonwhite racial subgroups at all in-clinic visits.

Overall, crisaborole-treated patients showed greater improvements in QoL than did vehicle-treated patients across race and ethnicity groups (Fig. 4). Crisaborole-treated patients aged $2-15$ years exhibited a greater reduction in CDLQI score than vehicle-treated patients at day 29 in all race and ethnicity groups (nominal $p<0.01$ ). Crisaboroletreated patients aged $\geq 16$ years showed a greater reduction in DLQI score than vehicle-treated patients at day 29 in the nonwhite, Hispanic/Latino, and not Hispanic/Latino groups (nominal $p<0.05$, Fig. 4), although no difference was observed for the white group (nominal $p=0.3948$ ). Parents/caregivers/families of crisaborole-treated patients aged 2-17 years showed a greater reduction in DFI score than families of vehicle-treated patients at day 29 in the white, nonwhite, and not Hispanic/Latino groups (nominal $p<0.05$, Fig. 4), although no difference was observed for the Hispanic/Latino group (nominal $p=0.2227$ ). Table 4 of the ESM reports CDLQI, DLQI, and DFI data for nonwhite racial subgroups at day 29.

\subsection{Safety Endpoints}

\subsubsection{Studies AD-301 and AD-302}

The safety population of the pivotal studies included a total of 1511 patients (Table 2). TEAEs (all causalities) were reported in $27.2-35.1 \%$ of crisaborole-treated patients and $25.0-34.3 \%$ of vehicle-treated patients across race and ethnicity. The majority of treatment-related TEAEs were mild or moderate. The most frequently reported treatment-related TEAE for all race and ethnicity groups was application site pain (crisaborole vs. vehicle): white [4.9\% $(n=30)$ vs. $1.3 \%$ $(n=4)$ ], nonwhite [3.8\% $(n=15)$ vs. $1.0 \%(n=2)]$, Hispanic/ 

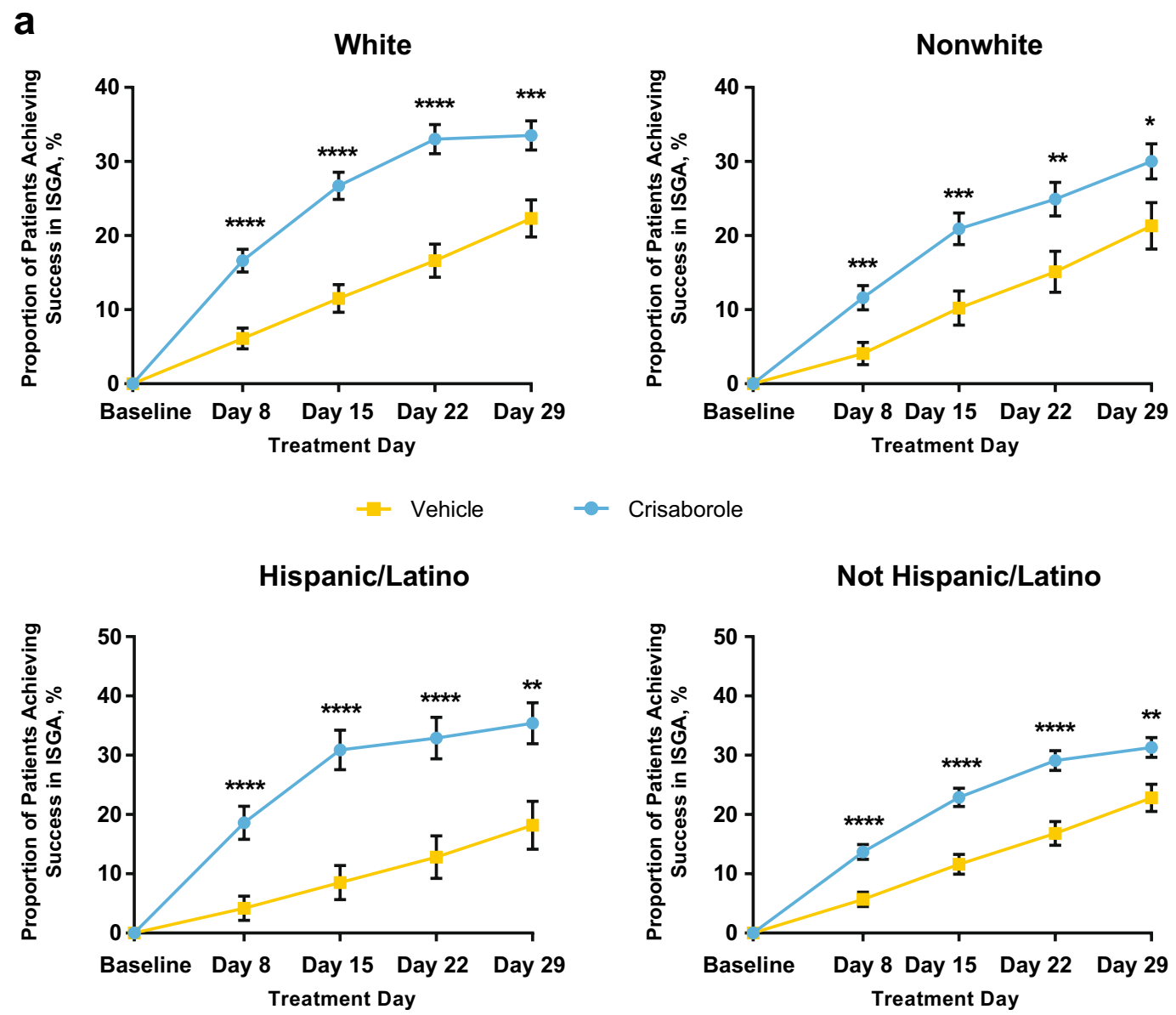

Crisaborole

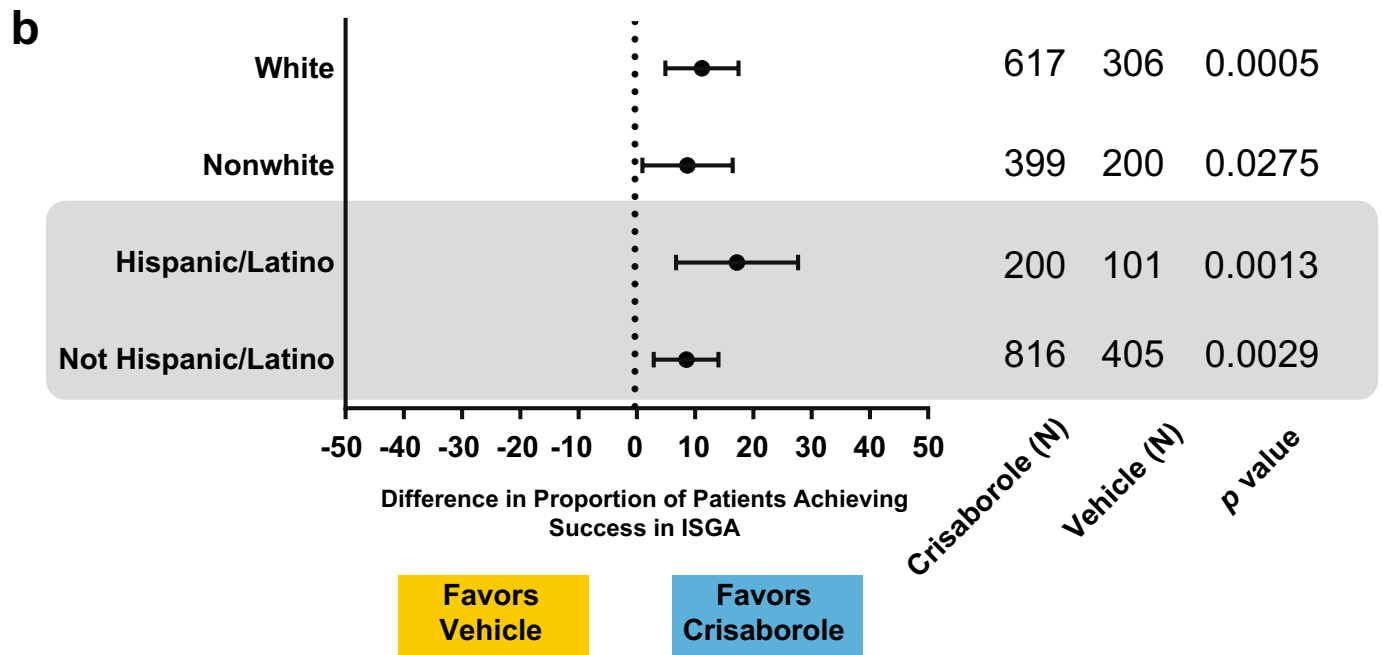

Fig. 1 Improvement in global disease severity: Investigator's Static Global Assessment (ISGA) success (AD-301 and AD-302, intentionto-treat [ITT] population). a Proportion of patients who achieved success in ISGA by ethnicity and race. Data are shown as mean \pm stand-
Not Hispanic/Latino

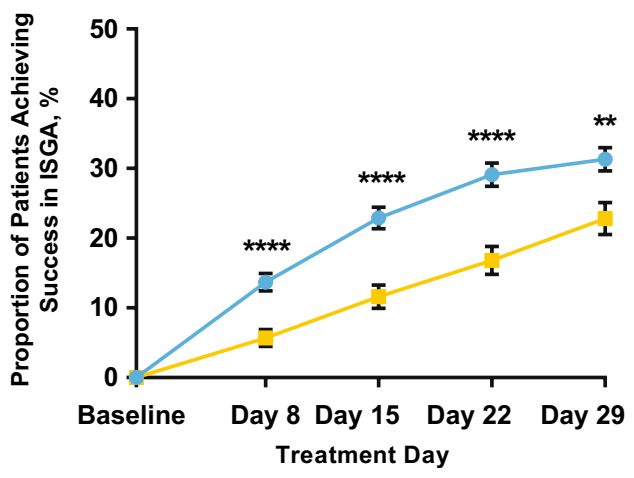

ard error of the mean. b Difference in the proportion of patients who achieved success in ISGA at day 29 by ethnicity and race. Data are shown as mean difference $\pm 95 \%$ confidence interval. Nominal $* p<0.05, * * p<0.01, * * * p<0.001, * * * * p<0.0001$ 

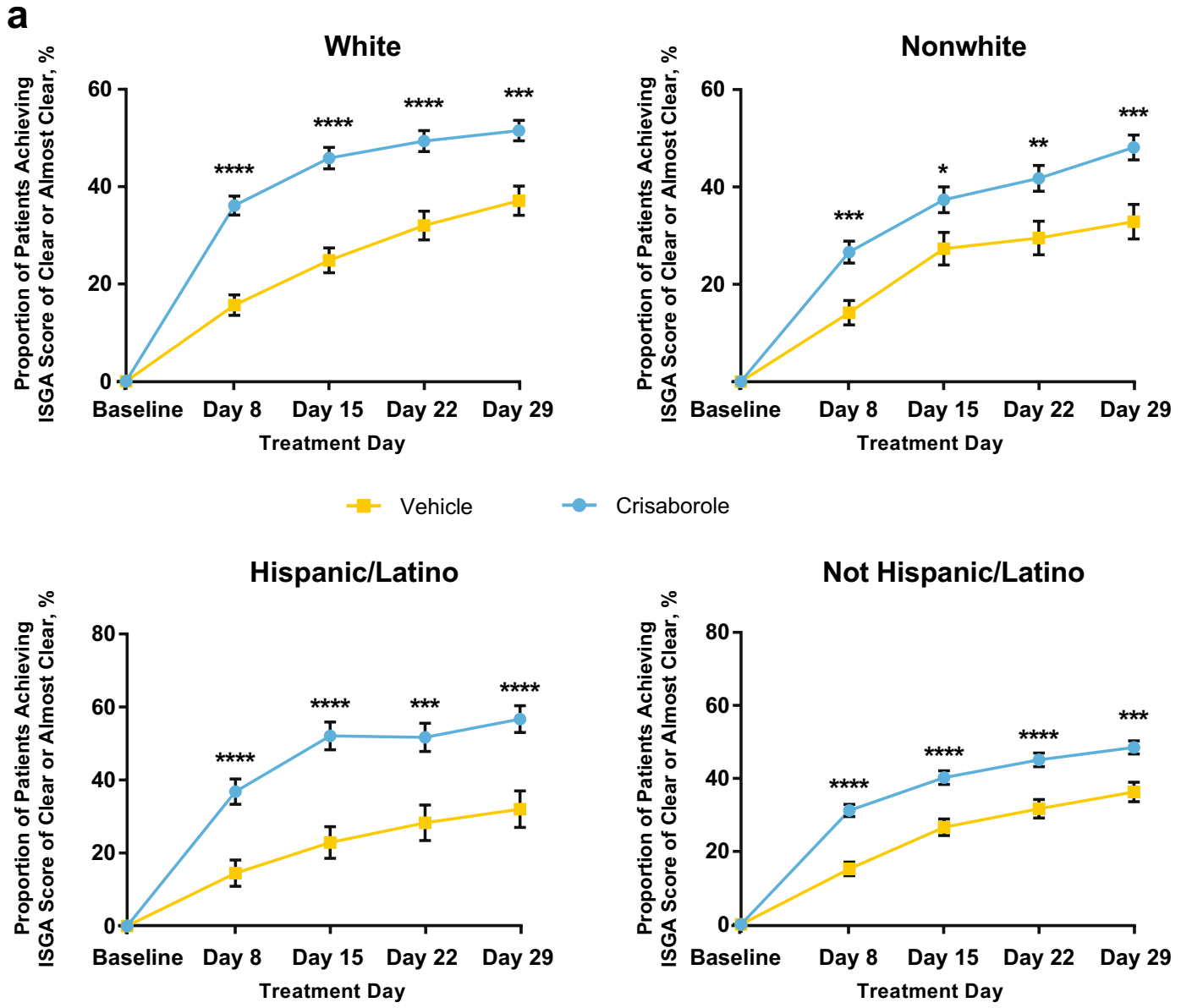

Crisaborole

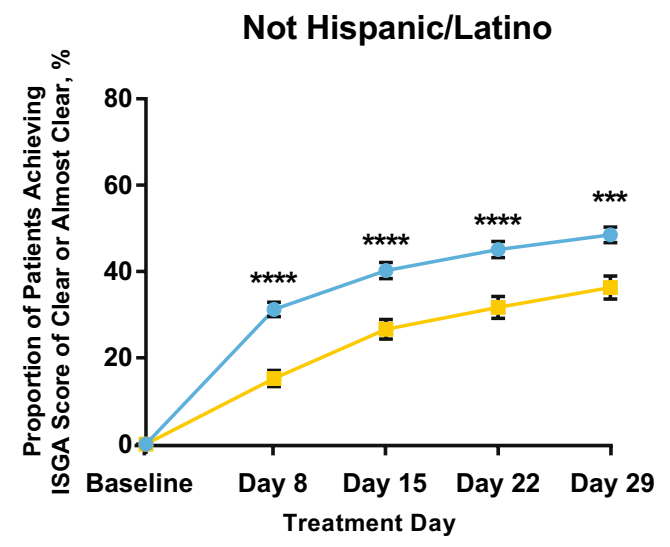

b

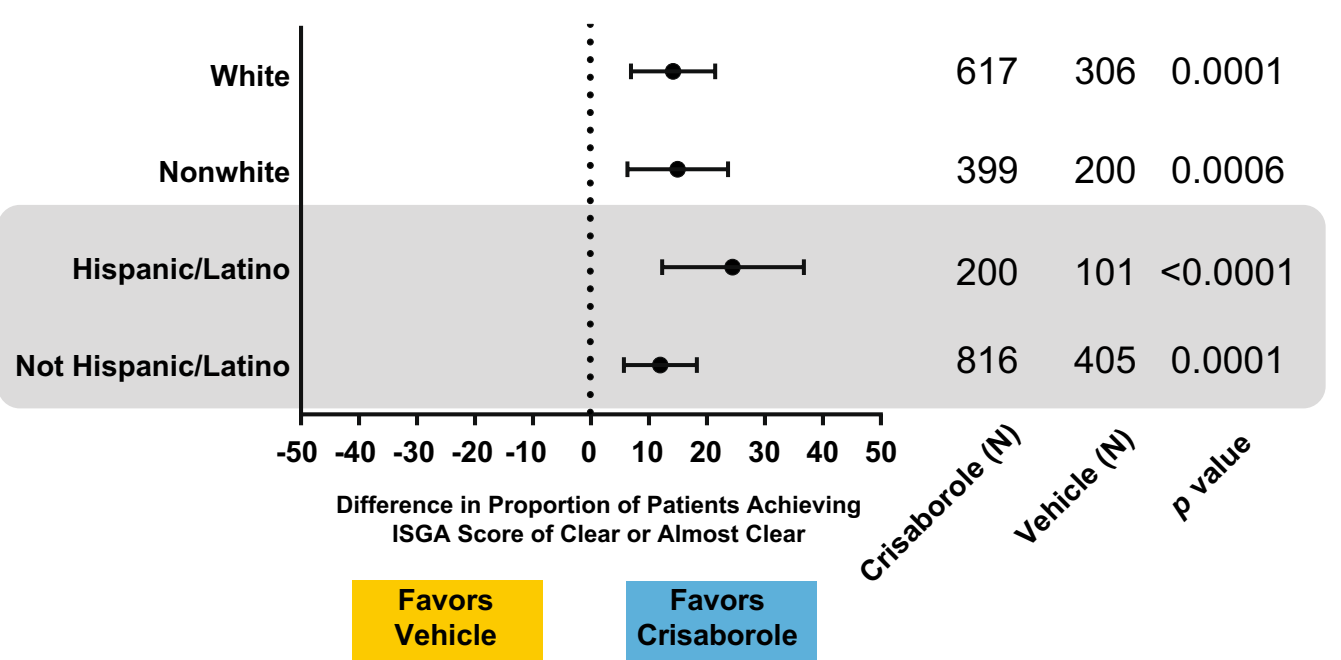

Fig. 2 Improvement in global disease severity: Investigator's Static Global Assessment (ISGA) score of clear or almost clear (AD-301 and AD-302, intention-to-treat [ITT] population). a Proportion of patients who achieved an ISGA score of clear (0) or almost clear (1) by ethnicity and race. Data are shown as mean \pm standard error of the mean. b Difference in the proportion of patients who achieved an ISGA score of clear (0) or almost clear (1) at day 29 by ethnicity and race. Data are shown as mean difference $\pm 95 \%$ confidence interval. Nominal $* p<0.05, * * p<0.01, * * * p<0.001, * * * * p<0.0001$ 

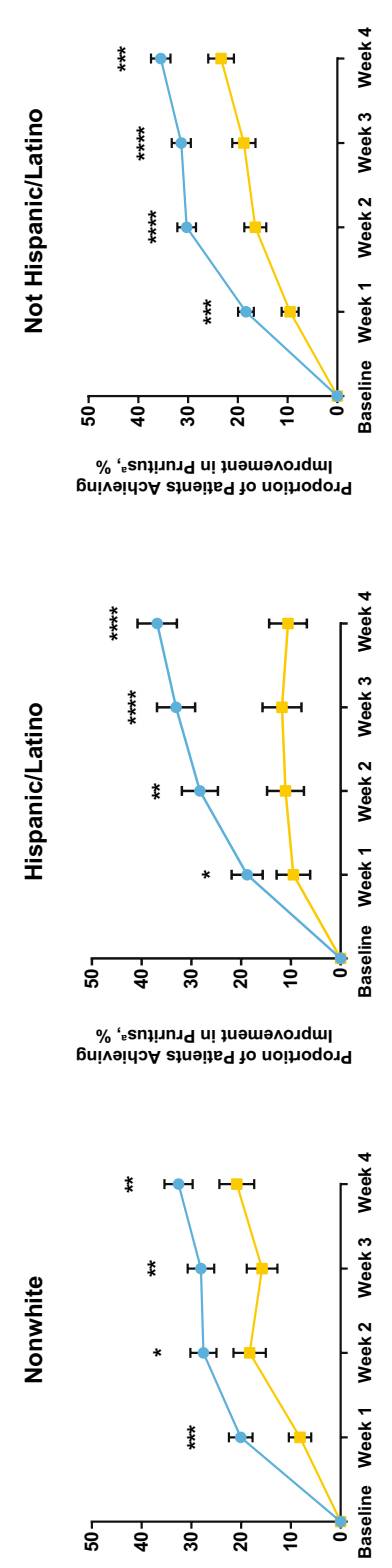

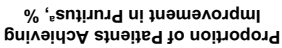

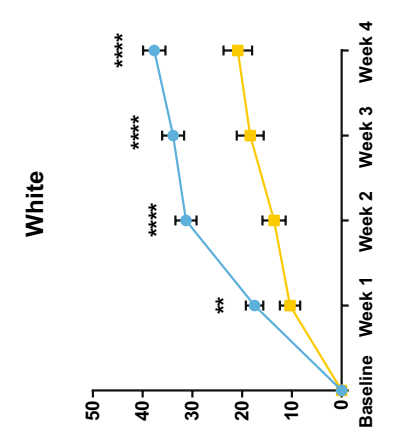

10

$\%$ 'snyunsd uा juamarosdup

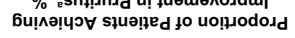
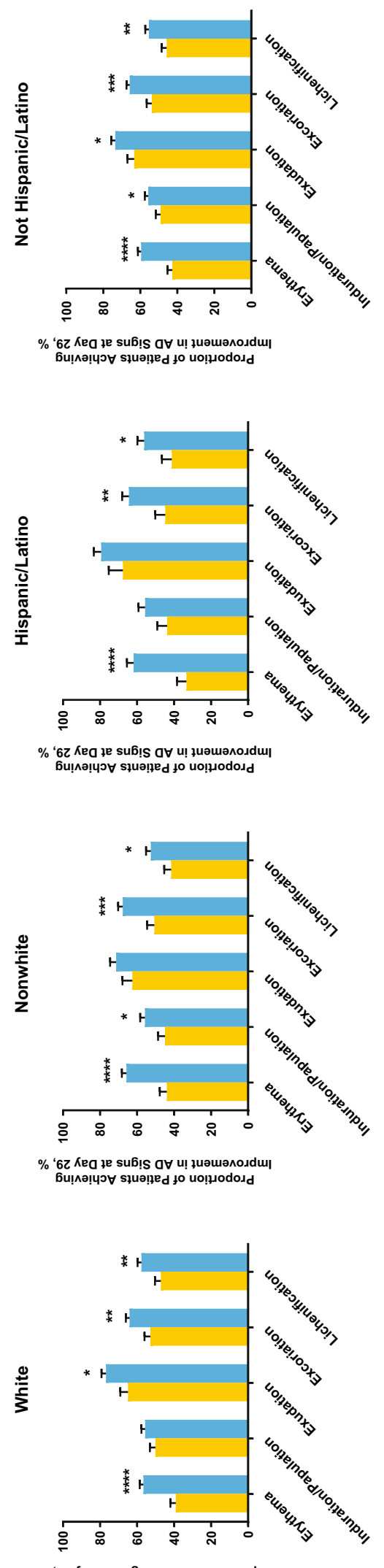

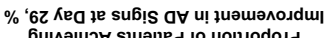

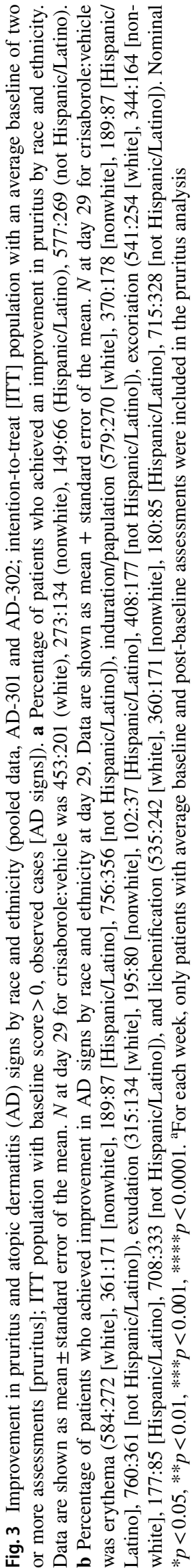




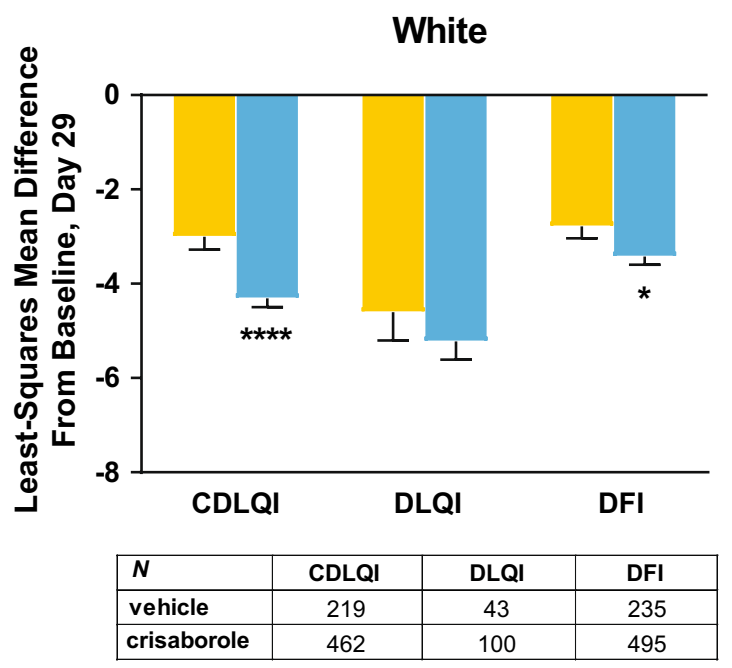

Vehicle

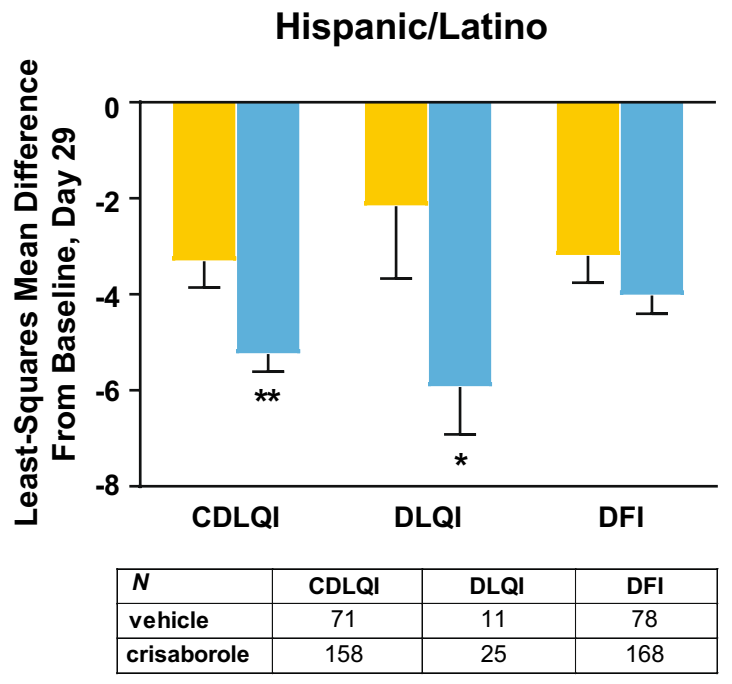

Fig. 4 Improvement in quality of life (QoL) of patients and parents/ caregivers at day 29, assessed as the least-squares mean change from baseline (pooled data, AD-301 and AD-302, intention-to-treat [ITT] population). Least-squares mean difference from baseline in QoL score by race and ethnicity. Data are shown as mean - standard

Latino [6.0\% ( $n=12)$ vs. $2.0 \%(n=2)$ ], not Hispanic/Latino [4.1\% $(n=33)$ vs. $1.0 \%(n=4)]$. One case of mild application site discoloration (hyperpigmentation) was reported in a white, not Hispanic/Latino crisaborole-treated patient. Rates of drug discontinuations due to TEAEs were similar across treatments and racial and ethnic groups (crisaborole vs. vehicle): white $[1.0 \%(n=6)$ vs. $2.0 \%(n=6)]$; nonwhite $[1.8 \%$ $(n=7)$ vs. $0.5 \%(n=1)]$, Hispanic/Latino $[1.0 \%(n=2)$ vs. $1.0 \%(n=1)]$, not Hispanic/Latino [1.4\% $(n=11)$ vs. $1.5 \%$ $(n=6)]$.

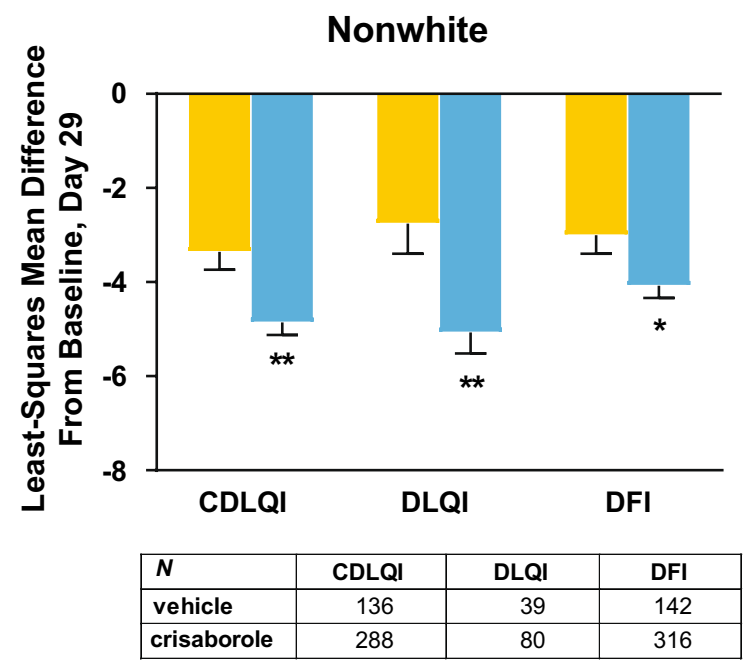

\section{Crisaborole}

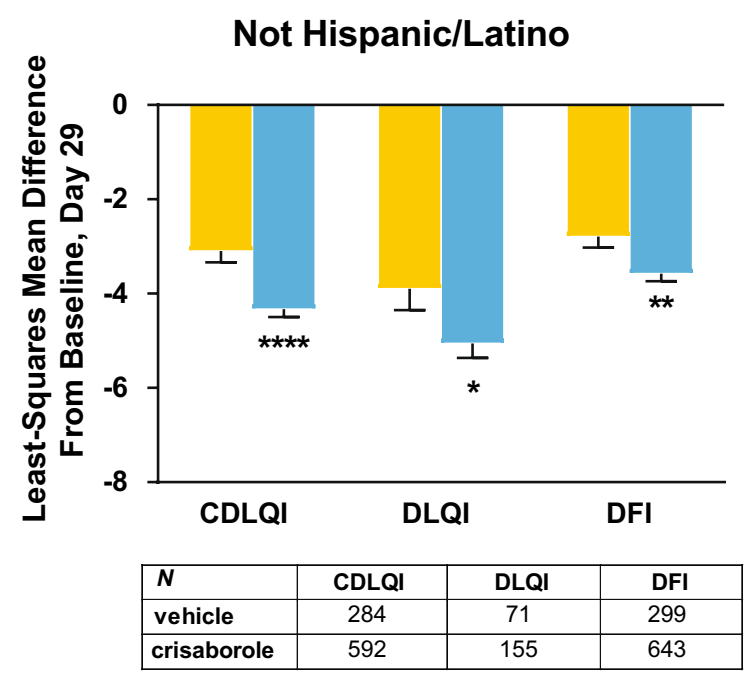

error of the mean. The number of patients achieving an improvement in QoL for each scale is listed in a table below each graph. CDLQI Children's Dermatology Life Quality Index, DLQI Dermatology Life Quality Index, DFI Dermatology Family Impact Questionnaire, nominal $* p<0.05, * * p<0.01, * * * p<0.001, * * * * p<0.0001$

As in the combined nonwhite group, application site pain was the most frequent treatment-related TEAE in nonwhite subgroups (crisaborole vs. vehicle): Asian/native Hawaiian/ other Pacific Islander [3.4\% $(n=2)$ vs. $2.9 \%(n=1)]$; black/ African American [2.8\% $(n=8)$ vs. $0 \%(n=0)]$; and other/ American Indian/Alaskan native $[9.1 \%(n=5)$ vs. $4.0 \%$ $(n=1)]$. Frequencies of TEAEs, serious TEAEs, treatmentrelated TEAEs, and drug discontinuations due to TEAEs in these subgroups are reported in Table 5 of the ESM. 
Table 2 Treatment-emergent adverse events (TEAEs) by race and ethnicity (pooled data, AD-301 and AD-302, safety population)

\begin{tabular}{|c|c|c|c|c|c|c|c|c|}
\hline \multirow{2}{*}{$\begin{array}{l}\text { Frequency of } \\
\text { TEAEs, } n(\%)\end{array}$} & \multicolumn{2}{|l|}{ White } & \multicolumn{2}{|l|}{ Nonwhite } & \multicolumn{2}{|c|}{ Hispanic/Latino } & \multicolumn{2}{|c|}{ Not Hispanic/Latino } \\
\hline & $\begin{array}{l}\text { Crisaborole } \\
N=615\end{array}$ & $\begin{array}{l}\text { Vehicle } \\
N=303\end{array}$ & $\begin{array}{l}\text { Crisaborole } \\
N=397\end{array}$ & $\begin{array}{l}\text { Vehicle } \\
N=196\end{array}$ & $\begin{array}{l}\text { Crisaborole } \\
N=199\end{array}$ & $\begin{array}{l}\text { Vehicle } \\
N=99\end{array}$ & $\begin{array}{l}\text { Crisaborole } \\
N=813\end{array}$ & $\begin{array}{l}\text { Vehicle } \\
N=400\end{array}$ \\
\hline$\geq 1$ TEAE & $216(35.1)$ & $96(31.7)$ & $108(27.2)$ & $49(25.0)$ & $67(33.7)$ & $34(34.3)$ & $260(32.0)$ & $111(27.8)$ \\
\hline $\begin{array}{l}\geq 1 \text { serious } \\
\text { TEAE }\end{array}$ & $4(0.7)$ & $1(0.3)$ & $4(1.0)$ & 0 & $3(1.5)$ & $1(1.0)$ & $5(0.6)$ & 0 \\
\hline $\begin{array}{l}\text { Any treatment- } \\
\text { related TEAE }\end{array}$ & $44(7.2)$ & $19(6.3)$ & $31(7.8)$ & $6(3.1)$ & $17(8.5)$ & $8(8.1)$ & $58(7.1)$ & $17(4.3)$ \\
\hline \multicolumn{9}{|c|}{ Treatment-related TEAEs } \\
\hline Mild & $19(3.1)$ & $7(2.3)$ & $13(3.3)$ & $3(1.5)$ & $10(5.0)$ & $2(2.0)$ & $22(2.7)$ & $8(2.0)$ \\
\hline Moderate & $18(2.9)$ & $9(3.0)$ & $14(3.5)$ & $3(1.5)$ & $5(2.5)$ & $5(5.1)$ & $27(3.3)$ & $7(1.8)$ \\
\hline Severe & $7(1.1)$ & $3(1.0)$ & $4(1.0)$ & 0 & $2(1.0)$ & $1(1.0)$ & $9(1.1)$ & $2(0.5)$ \\
\hline
\end{tabular}

The safety population was defined as all patients who were randomized, received $\geq 1$ confirmed dose of the study drug, and had $\geq 1$ post-baseline assessment

Table 3 Treatment-emergent adverse events (TEAEs) by race and ethnicity (AD-303, safety population)

\begin{tabular}{|c|c|c|c|c|}
\hline Frequency of TEAEs, $n(\%)$ & $\begin{array}{l}\text { White } \\
N=315\end{array}$ & $\begin{array}{l}\text { Nonwhite } \\
N=202\end{array}$ & $\begin{array}{l}\text { Hispanic/Latino } \\
N=82\end{array}$ & $\begin{array}{l}\text { Not Hispanic/Latino } \\
N=435\end{array}$ \\
\hline$\geq 1$ TEAE & $226(71.7)$ & $109(54.0)$ & $57(69.5)$ & $279(64.1)$ \\
\hline$\geq 1$ serious TEAE & $3(1.0)$ & $6(3.0)$ & $2(2.4)$ & $7(1.6)$ \\
\hline Any treatment-related TEAE & $33(10.5)$ & $20(9.9)$ & $12(14.6)$ & $41(9.4)$ \\
\hline \multicolumn{5}{|l|}{ Treatment-related TEAEs } \\
\hline Mild & $13(4.1)$ & $6(3.0)$ & $3(3.7)$ & $16(3.7)$ \\
\hline Moderate & $16(5.1)$ & $11(5.4)$ & $6(7.3)$ & $21(4.8)$ \\
\hline Severe & $4(1.3)$ & $3(1.5)$ & $3(3.7)$ & $4(0.9)$ \\
\hline
\end{tabular}

TEAEs were those with an onset on or after the day of the first study drug (crisaborole ointment, 2\%, or vehicle) application in AD-301 or AD-302; includes all TEAEs with an onset on or after the day of the first study drug application (crisaborole or vehicle) in AD-301 or AD-302; the safety population was defined as all patients who were randomized, received $\geq 1$ confirmed dose of study drug, and had $\geq 1$ post-baseline assessment

\subsubsection{Study AD-303}

Following completion of the pivotal studies, 517 patients enrolled in the long-term safety study (Table 3). TEAEs (all causalities, across pivotal and extension studies) were reported in $54.0-71.7 \%$ of crisaborole-treated patients across races and ethnicities. The majority of treatment-related TEAEs were mild or moderate. The most frequently reported treatment-related TEAEs in white and nonwhite patients were $\mathrm{AD}$ [white: $3.2 \%(n=10)$; nonwhite: $3.0 \%(n=6)$ ] and application site pain [white: $2.9 \%(n=9)$; nonwhite: $1.5 \%$ $(n=3)]$. AD events involved worsening, exacerbation, flare, or flare-up of existing AD. The most frequently reported treatment-related TEAEs in Hispanic/Latino patients were AD [7.3\% $(n=6)]$ and application site infection [2.4\% $(n=2)]$. In not Hispanic/Latino patients, application site pain [2.5\% $(n=11)]$ and $\mathrm{AD}[2.3 \%(n=10)]$ were most frequently reported. Mean duration of application site pain in patients enrolled in the long-term safety study was 8.3 days (white, $n=10$ ), 26.0 days (nonwhite, $n=2$ ), 1.0 days (Hispanic/ Latino, $n=1$ ), and 12.2 days (not Hispanic/Latino, $n=11$ ). The proportion of application site pain events that resolved within 1 day was $40.0 \%$ (white, 4/10), $0 \%$ (nonwhite, $0 / 2$ ), 100.0\% (Hispanic/Latino, 1/1), and 27.3\% (not Hispanic/ Latino, 3/11). Rates of drug discontinuations due to TEAEs were similar across race and ethnicity groups: white [1.6\% $(n=5)]$, nonwhite [2.0\% $(n=4)]$, Hispanic/Latino [3.7\% $(n=3)]$, not Hispanic/Latino [1.4\% $(n=6)]$. Application site pain was the reason for discontinuation in two not Hispanic/ Latino patients $(0.5 \%)$, one white patient $(0.3 \%)$, and one nonwhite patient $(0.5 \%)$.

Frequency of TEAEs, serious TEAEs, treatment-related TEAEs, and drug discontinuations due to TEAEs in nonwhite racial subgroups in the long-term safety extension study are reported in Table 6 of the ESM. As in the combined nonwhite group, $\mathrm{AD}$ was the most frequently reported 
treatment-related TEAE in Asian/native Hawaiian/other Pacific Islander patients (3.4\%, $n=1)$, black/African American patients $(2.0 \%, n=3)$, and other/American Indian/Alaskan native patients $(9.5 \%, n=2)$. Application site pain was reported in two black/African American patients (1.3\%) and one other/American Indian/Alaskan native patient (4.8\%). Application site infection was reported in two black/African American patients (1.3\%).

\section{Discussion}

This pooled post hoc analysis evaluated the safety and efficacy of crisaborole according to race and ethnicity. We found that crisaborole was efficacious for the treatment of patients aged $\geq 2$ years with mild-to-moderate $A D$ regardless of racial or ethnic group. The safety profile of crisaborole was consistent across races and ethnicities, with a low frequency of treatment-related TEAEs in the pivotal and longterm safety extension studies.

The efficacy of crisaborole in decreasing disease severity and improving AD signs and QoL across all racial and ethnic groups indicates that it may have the potential to lessen the burden of disease in patients with skin of color. Available data suggest a higher burden of $A D$ in patients with skin of color. An analysis of two national ambulatory care databases found that black and Asian children were two to three times more likely to visit a physician or hospital for a diagnosis of AD than white children during the survey period (1997-2004) [5]. An earlier study reported a similar pattern in ambulatory care visits, finding that despite lower overall healthcare utilization, black and Asian/Pacific Islander children were three to seven times more likely to visit a physician for AD than white children during the survey period (1990-8) [8]. A study in UK children with $\mathrm{AD}$ found that while white and black children evaluated with the SCORing AD (SCORAD) index initially showed no difference in disease severity, when erythema was excluded from severity scores, black children were nearly six times more likely to have severe AD than white children [10]. An increased likelihood of severe $\mathrm{AD}$ has consequences for disease burden, as $\mathrm{AD}$ severity is correlated with a greater number of chronic health conditions, poorer overall health, higher degree of sleep impairment, and greater use of health services [25]. In the present study, we observed improvements in global disease severity and pruritus across all racial and ethnic groups. Crisaborole improved AD signs that are common in patients with skin of color, including induration/ papulation (improved by crisaborole in nonwhite patients) and lichenification (improved by crisaborole in nonwhite and Hispanic/Latino patients). Significant differences vs. vehicle in the proportions of patients achieving improvement in lichenification were observed at all time points in Hispanic/Latino patients and at three of the four time points in nonwhite patients.

The safety profile of crisaborole suggests that it may be an appropriate treatment option for patients with skin of color. Hyperpigmentation is a concerning $\mathrm{AE}$ in patients with skin of color, who are more likely to experience dyspigmentation in healed $\mathrm{AD}$ lesions $[9,13]$. While the risk of AEs can be reduced through selection of appropriate topical corticosteroid potency and treatment duration [18, 26], long-term topical corticosteroid use may be associated with dyspigmentation and skin atrophy, including bruising and striae [27-29]. In these phase III studies of crisaborole, mild treatment-related application site discoloration was reported in one white, not Hispanic/Latino crisaborole-treated patient who experienced gray skin discoloration of hands and feet at the application site. A larger difference from the vehicle in rates of treatment-related TEAEs was observed in nonwhite and not Hispanic/Latino groups compared with the white and Hispanic/Latino groups in the pivotal trials (AD-301 and $\mathrm{AD}-302$ ); however, this difference was largely driven by a reduction in the rate of treatment-related TEAEs among vehicle-treated patients in nonwhite and not Hispanic/Latino groups. The difference in vehicle-related TEAEs may reflect differences in dermal reaction to vehicle components, the smaller sample size of the vehicle group resulting from the 2:1 randomization scheme, or limitations of the post hoc analysis. Nevertheless, prospective clinical trials are needed to further assess the efficacy and safety of topical therapies in patients with $\mathrm{AD}$ by race and ethnicity.

There are limitations to this study. As this was a post hoc analysis and therefore not powered for subgroup comparison, interpretation of the significance of results should not be based on nominal $p$ values alone, especially for comparisons of nonwhite racial subgroups because of the small sample size. Furthermore, the present study did not assess skin tone using standardized methods, such as Fitzpatrick scoring, potentially leading to inconsistency in the reporting of race or ethnicity in some patients.

\section{Conclusions}

Regardless of race or ethnicity, crisaborole improved global disease severity in patients aged $\geq 2$ years with mild-to-moderate $\mathrm{AD}$. Crisaborole was efficacious in reducing $\mathrm{AD}$ signs common in patients with skin of color and was associated with a low frequency of treatment-related TEAEs, suggesting that crisaborole may be an appropriate treatment option for these patients. 
Acknowledgements We thank the study patients, investigators, and investigational sites whose participation made these studies possible. Medical writing and editorial assistance under the guidance of the authors was provided by Madeline L. Pfau, PhD, and Corey Mandel, $\mathrm{PhD}$, of ApotheCom, San Francisco, CA, USA and was funded by Pfizer Inc. in accordance with Good Publication Practice (GPP3) guidelines (Ann Intern Med. 2015;163:461-4).

\section{Compliance with Ethical Standards}

Funding This study was funded by Pfizer Inc.

Conflict of interest Valerie D. Callender is an advisor and speaker for Pfizer. Andrew F. Alexis has received grants from Galderma, Novartis, Almirall, RXi Pharmaceuticals, Bristol-Myers Squibb, Celgene, Menlo, and LEO Pharma and consulting fees or honoraria from Pfizer, Novartis, Almirall, Menlo, Trevi, Dermavant, Unilever, Celgene, BiopharmX, Cipla, Beiersdorf, Valeant, Galderma, Sanofi-Genzyme, and LEO Pharma. Andrew F. Alexis has also received writing assistance, medicines, equipment, or administrative support from Pfizer and payment for lectures from Pfizer and LEO Pharma. Linda F. Stein Gold has received grants from Pfizer, LEO Pharma, and Incyte, and payment for lectures from Pfizer and LEO Pharma. Mark G. Lebwohl is an employee of Mount Sinai and has received research funds from AbbVie, Boehringer Ingelheim, Celgene, Eli Lilly, Incyte, Janssen/Johnson \& Johnson, LEO Pharma, MedImmune/AstraZeneca, Novartis, Pfizer, SCIderm, Valeant, and Vidac Pharma. Mark G. Lebwohl is also a consultant for Allergan, Aqua Pharmaceuticals, Boehringer Ingelheim, LEO Pharma, Menlo, Mitsubishi, Promius, and Verrica. Amy S. Paller has been an investigator for AbbVie, AnaptysBio, Eli Lilly, Galderma, Incyte, LEO Pharma, Janssen, Novartis, and Sanofi-Regeneron, and has been a consultant with honoraria for AbbVie, Amgen, Asana BioSciences, Dermavant, Dermira, Galderma, Eli Lilly, Forte, LEO Pharma, Matrisys Bioscience, Menlo, MorphoSys/Galapagos, Novartis, Pfizer, and Sanofi-Regeneron. Seemal R. Desai has received consulting fees or honoraria and payment for lectures from Pfizer. Huaming Tan, William C. Ports, and Michael A. Zielinski are employees and stockholders of Pfizer Inc. Anna M. Tallman was an employee and stockholder of Pfizer Inc. at the time of the analysis and initial manuscript development.

Ethics approval All study procedures involving human participants were performed in accordance with all applicable laws, rules, and regulations, including the principles of Good Clinical Practice and the ethical principles outlined in the 1964 Helsinki Declaration and its later amendments.

Consent to participate Informed consent was obtained from all study participants, and the institutional review board of each investigational center approved all study protocols, consent forms, and relevant supporting data. This article does not contain any studies with animals performed by any of the authors.

Data availability Upon request, and subject to certain criteria, conditions and exceptions (see https://www.pfizer.com/science/clinical-trials/ trial-data-and-results for more information), Pfizer will provide access to individual de-identified participant data from Pfizer-sponsored global interventional clinical studies conducted for medicines, vaccines, and medical devices (1) for indications that have been approved in the United States and/or European Union or (2) in programs that have been terminated (i.e., development for all indications has been discontinued). Pfizer will also consider requests for the protocol, data dictionary, and statistical analysis plan. Data may be requested from Pfizer trials 24 months after study completion. The de-identified par- ticipant data will be made available to researchers whose proposals meet the research criteria and other conditions, and for which an exception does not apply, via a secure portal. To gain access, data requestors must enter into a data access agreement with Pfizer.

Open Access This article is distributed under the terms of the Creative Commons Attribution-NonCommercial 4.0 International License (http://creativecommons.org/licenses/by-nc/4.0/), which permits any noncommercial use, distribution, and reproduction in any medium, provided you give appropriate credit to the original author(s) and the source, provide a link to the Creative Commons license, and indicate if changes were made.

\section{References}

1. Bieber T. Atopic dermatitis. N Engl J Med. 2008;358(14):1483-94.

2. Bieber T. Atopic dermatitis. Ann Dermatol. 2010;22(2):125-37.

3. Schachner L, Ling NS, Press S. A statistical analysis of a pediatric dermatology clinic. Pediatr Dermatol. 1983;1(2):157-64.

4. Shaw TE, Currie GP, Koudelka CW, Simpson EL. Eczema prevalence in the United States: data from the 2003 National Survey of Children's Health. J Investig Dermatol. 2011;131(1):67-73.

5. Horii KA, Simon SD, Liu DY, Sharma V. Atopic dermatitis in children in the United States, 1997-2004: visit trends, patient and provider characteristics, and prescribing patterns. Pediatrics. 2007;120(3):e527-34.

6. Baker RP. Incidence of atopic dermatitis and eczema by ethnic group seen within a general pediatric practice. Perm J. 1999;3(1):31-2.

7. Odhiambo JA, Williams HC, Clayton TO, Robertson CF, Asher MI. Global variations in prevalence of eczema symptoms in children from ISAAC phase three. J Allergy Clin Immunol. 2009;124(6):1251-8.

8. Janumpally SR, Feldman SR, Gupta AK, Fleischer AB Jr. In the United States, blacks and Asian/Pacific Islanders are more likely than whites to seek medical care for atopic dermatitis. Arch Dermatol. 2002;138(5):634-7.

9. Vachiramon V, Tey HL, Thompson AE, Yosipovitch G. Atopic dermatitis in African American children: addressing unmet needs of a common disease. Pediatr Dermatol. 2012;29(4):395-402.

10. Ben-Gashir MA, Hay RJ. Reliance on erythema scores may mask severe atopic dermatitis in black children compared with their white counterparts. Br J Dermatol. 2002;147(5):920-5.

11. Mei-Yen Yong A, Tay YK. Atopic dermatitis: racial and ethnic differences. Dermatol Clin. 2017;35(3):395-402.

12. Noda S, Suarez-Farinas M, Ungar B, Kim SJ, de Guzman Strong $\mathrm{C}, \mathrm{Xu} \mathrm{H}$, et al. The Asian atopic dermatitis phenotype combines features of atopic dermatitis and psoriasis with increased TH17 polarization. J Allergy Clin Immunol. 2015;136(5):1254-64.

13. Kaufman BP, Guttman-Yassky E, Alexis AF. Atopic dermatitis in diverse racial and ethnic groups: variations in epidemiology, genetics, clinical presentation and treatment. Exp Dermatol. 2018;27(4):340-57.

14. Sanyal RD, Pavel AB, Glickman J, Chan TC, Zheng X, Zhang $\mathrm{N}$, et al. Atopic dermatitis in African American patients is TH2/ TH22-skewed with TH1/TH17 attenuation. Ann Allergy Asthma Immunol. 2019;122(1):99-110.

15. Grundbacher FJ. Causes of variation in serum $\operatorname{IgE}$ levels in normal populations. J Allergy Clin Immunol. 1975;56(2):104-11.

16. Sueki H, Whitaker-Menezes D, Kligman AM. Structural diversity of mast cell granules in black and white skin. Br J Dermatol. 2001;144(1):85-93. 
17. Hirano SA, Murray SB, Harvey VM. Reporting, representation, and subgroup analysis of race and ethnicity in published clinical trials of atopic dermatitis in the United States between 2000 and 2009. Pediatr Dermatol. 2012;29(6):749-55.

18. Eichenfield LF, Tom WL, Berger TG, Krol A, Paller AS, Schwarzenberger K, et al. Guidelines of care for the management of atopic dermatitis: section 2. Management and treatment of atopic dermatitis with topical therapies. J Am Acad Dermatol. 2014;71(1):116-32.

19. Schneider L, Tilles S, Lio P, Boguniewicz M, Beck L, LeBovidge J, et al. Atopic dermatitis: a practice parameter update 2012. J Allergy Clin Immunol. 2013;131(2):295-9.

20. Sidbury R, Davis DM, Cohen DE, Cordoro KM, Berger TG, Bergman JN, et al. Guidelines of care for the management of atopic dermatitis: section 3. Management and treatment with phototherapy and systemic agents. J Am Acad Dermatol. 2014;71(2):327-49.

21. Jarnagin K, Chanda S, Coronado D, Ciaravino V, Zane LT, Guttman-Yassky E, et al. Crisaborole topical ointment, 2\%: a nonsteroidal, topical, anti-inflammatory phosphodiesterase 4 inhibitor in clinical development for the treatment of atopic dermatitis. J Drugs Dermatol. 2016;15(4):390-6.

22. Paller AS, Tom WL, Lebwohl MG, Blumenthal RL, Boguniewicz M, Call RS, et al. Efficacy and safety of crisaborole ointment, a novel, nonsteroidal phosphodiesterase 4 (PDE4) inhibitor for the topical treatment of atopic dermatitis (AD) in children and adults. J Am Acad Dermatol. 2016;75(3):494-503.
23. Eichenfield LF, Call RS, Forsha DW, Fowler J Jr, Hebert AA, Spellman M, et al. Long-term safety of crisaborole ointment $2 \%$ in children and adults with mild to moderate atopic dermatitis. J Am Acad Dermatol. 2017;77(4):641-9.

24. Yosipovitch G, Gold LF, Lebwohl MG, Silverberg JI, Tallman AM, Zane LT. Early relief of pruritus in atopic dermatitis with crisaborole ointment, a non-steroidal, phosphodiesterase 4 inhibitor. Acta Derm Venereol. 2018;98(5):484-9.

25. Silverberg JI, Simpson EL. Associations of childhood eczema severity: a US population-based study. Dermatitis. 2014;25(3):107-14.

26. Korting HC, Unholzer A, Schafer-Korting M, Tausch I, Gassmueller J, Nietsch KH. Different skin thinning potential of equipotent medium-strength glucocorticoids. Skin Pharmacol Appl Skin Physiol. 2002;15(2):85-91.

27. Hengge UR, Ruzicka T, Schwartz RA, Cork MJ. Adverse effects of topical glucocorticosteroids. J Am Acad Dermatol. 2006;54(1):1-15 (quiz 6-8).

28. Barnes L, Kaya G, Rollason V. Topical corticosteroid-induced skin atrophy: a comprehensive review. Drug Saf. 2015;38(5):493-509.

29. Aschoff R, Schmitt J, Knuschke P, Koch E, Brautigam M, Meurer M. Evaluation of the atrophogenic potential of hydrocortisone $1 \%$ cream and pimecrolimus $1 \%$ cream in uninvolved forehead skin of patients with atopic dermatitis using optical coherence tomography. Exp Dermatol. 2011;20(10):832-6. 